\title{
LETTER
}

\section{Severe and early quadriceps weakness in mechanically ventilated patients}

\author{
Isabelle Vivodtzev ${ }^{1,2,3^{*}}$, Andrée-Anne Devost ${ }^{1}$, Didier Saey ${ }^{1}$, Sophie Villeneuve ${ }^{1}$, Geneviève Boilard ${ }^{1}$, \\ Philippe Gagnon ${ }^{1}$, Steeve Provencher ${ }^{1}$, Mathieu Simon ${ }^{1}$, Richard Baillot ${ }^{1}$, François Maltais ${ }^{1}$ and François Lellouche
}

ICU-acquired weakness has been reported in patients with prolonged mechanical ventilation [1], leading to prolonged weaning, poor quality of life after ICU discharge, and high ICU-related cost [2]. Muscle weakness is the primary manifestation of critical illness polyneuropathy or myopathy or both.

Although quadriceps strength has never been objectively quantified in the ICU, we previously evidenced quadriceps muscle weakness by using magnetic stimulation of the femoral nerve in patients with chronic obstructive pulmonary disease (COPD) and this non-invasive technique allows a non-effort-dependent assessment of quadriceps strength $[3,4]$. Thus, one objective of this pilot study was to evaluate the feasibility of assessing quadriceps strength by using this previously validated technique in sedated patients on mechanical ventilation at different stages after ICU admission using magnetic stimulation of the femoral nerve. The study was approved by the ethics committee of the Institut Universitaire de Cardiologie et de Pneumologie de Québec (CER20392). Signed informed consent was obtained from relatives for all patients.

Quadriceps twitch tension (Twq) assessment was performed in 13 consecutive sedated and mechanically ventilated patients with organ failure (Table 1). Twq measurements were repeated after awakening in nine patients. Mean Twq was $1.8 \pm 1.3 \mathrm{~kg}$ for the whole group of patients. As shown in Figure 1, Twq was two times lower in ICU patients than in COPD patients $(P<0.001)$ and four times lower than in healthy subjects $(P<0.001)$. Furthermore, there was no significant difference in Twq when patients were sedated or awake. The reproducibility between these two measurements was good (Figure 2).

\footnotetext{
* Correspondence: ivivodtzev@chu-grenoble.fr

${ }^{1}$ Centre de Recherche de I'Institut Universitaire de Cardiologie et de

Pneumologie de Québec, Université Laval, 2725 chemin Sainte-Foy, Québec G1V 4G5, Canada

${ }^{2}$ Univ Grenoble Alpes, Grenoble HP2 38000, France

Full list of author information is available at the end of the article
}

\section{Table 1 Patient characteristics at baseline}

\begin{tabular}{|c|c|}
\hline \multicolumn{2}{|l|}{ Demographics } \\
\hline Males/females, number & $8 / 5$ \\
\hline Age, years & $71 \pm 9$ \\
\hline Body mass index, $\mathrm{kg} / \mathrm{m}^{2}$ & $25 \pm 4$ \\
\hline \multicolumn{2}{|l|}{ Arterial blood gases } \\
\hline $\mathrm{PaO}_{2}, \mathrm{~mm} \mathrm{Hg}$ & $82 \pm 34$ \\
\hline $\mathrm{PaCO}_{2}, \mathrm{~mm} \mathrm{Hg}$ & $41 \pm 8$ \\
\hline $\mathrm{pH}$ & $7.43 \pm 0.08$ \\
\hline $\mathrm{SaO}_{2}$, percentage & $95 \pm 2$ \\
\hline \multicolumn{2}{|l|}{ ICU admission } \\
\hline Cardiac surgery ICU & $9(69 \%)$ \\
\hline Respiratory ICU & $4(31 \%)$ \\
\hline \multicolumn{2}{|l|}{ Comorbidities } \\
\hline COPD & $8(62 \%)$ \\
\hline Hypertension & $11(85 \%)$ \\
\hline Hypothyroid & $3(23 \%)$ \\
\hline Dyslipidemia & $8(62 \%)$ \\
\hline Diabetes mellitus & $4(31 \%)$ \\
\hline \multicolumn{2}{|l|}{ Risk factor for polyneuropathy } \\
\hline Mechanical ventilation more than 72 hours & $12(85 \%)$ \\
\hline Suboptimal glucose control ${ }^{a}$ & $11(85 \%)$ \\
\hline Steroids & $5(38 \%)$ \\
\hline Septic shock & $6(46 \%)$ \\
\hline Neuromuscular blocker & $5(38 \%)$ \\
\hline Risk factors, mean & $3 \pm 1$ \\
\hline Duration of hospitalization before strength assessment, days & $7 \pm 4$ \\
\hline Sedation condition, RASS score & $-3.8 \pm 1.5$ \\
\hline
\end{tabular}

Data are presented as mean \pm standard deviation or as number (percentage). ${ }^{a}$ Suboptimal glucose control is defined as repeated measurements of capillary or venous glucose measurements above $10 \mathrm{mmol} / \mathrm{L}$ (at least two consecutives). COPD, chronic obstructive pulmonary disease; $\mathrm{PaCO}_{2}$, arterial pressure in carbon dioxide; $\mathrm{PaO}_{2}$, arterial pressure in oxygen; RASS, Richmond Agitation-Sedation Scale; $\mathrm{SaO}_{2}$, arterial saturation in oxygen.

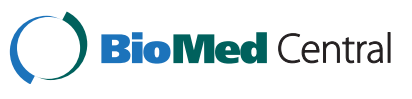

C 2014 Vivodtzev et al.; licensee BioMed Central Ltd. The licensee has exclusive rights to distribute this article, in any medium, for 6 months following its publication. After this time, the article is available under the terms of the Creative Commons Attribution License (http://creativecommons.org/licenses/by/4.0), which permits unrestricted use, distribution, and reproduction in any medium, provided the original work is properly cited. 


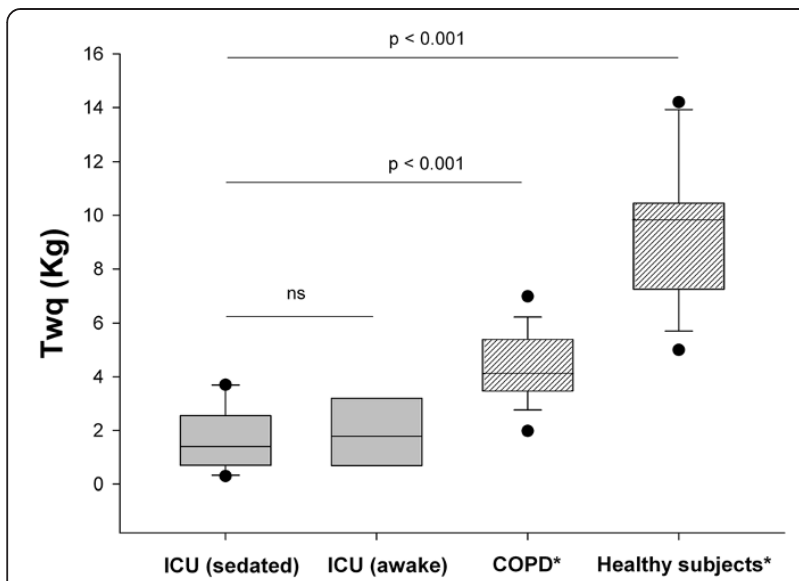

Figure 1 Quadriceps twitch tension (Twq) in ICU patients.

Stimulation was applied in ICU mechanical ventilation patients who were sedated $(n=13)$ or awake $(n=7)$ (grey), patients with age-related chronic obstructive pulmonary disease (COPD) $(n=18)$, and healthy subjects $(n=16)$ (shaded). The ends of the boxes define the 25th and 75th percentiles, and a line at the median and error bars define the 10th and 90th percentiles. *Previously measured in our laboratory [3]. ns, Not significant.

Strength measurements have been performed in patients during septic shock $(\mathrm{n}=2)$ or after a dialysis session $(\mathrm{n}=2)$, and a major reduction of muscle strength (Twq $<1 \mathrm{~kg}$ ) was observed in these circumstances.

Our results confirm the evidence of early severe muscle weakness in mechanically ventilated patients and show that measurement of muscle strength by magnetic stimulation of the femoral nerve may be useful in ICU patients, particularly for assessing recovery or the effect of therapeutic interventions, as previously suggested by Ginz and colleagues [5]. A noteworthy result is that some events (such as dialysis and sepsis) may modify the muscle strength and need to be considered when interpreting muscle strength data in this context. Our data showing that muscle weakness is an early process in the ICU favor early treatment to prevent rather than delay treatment to treat this condition.

\section{Abbreviations}

COPD: Chronic obstructive pulmonary disease; Twq: Quadriceps twitch tension.

\section{Competing interests}

The authors declare that they have no competing interests.

\section{Authors' contributions}

IV and FL contributed to the study concept and design, data analysis, the interpretation of results, and the writing of the manuscript. A-AD, DS, SV, $G B$, and PG participated in the recruitment of patients, data acquisition, and the writing of the manuscript. SP, MS, and RB participated in the recruitment of patients. FM contributed to the study concept and design and the writing of the manuscript. All authors read and approved the final manuscript.

\section{Acknowledgments}

Funding was provided by the Canadian Foundation for Innovation (FRSQ).

\section{Author details}

${ }^{1}$ Centre de Recherche de I'Institut Universitaire de Cardiologie et de Pneumologie de Québec, Université Laval, 2725 chemin Sainte-Foy, Québec G1V 4G5, Canada. ${ }^{2}$ Univ Grenoble Alpes, Grenoble HP2 38000, France. ${ }^{3}$ Inserm U 1042, Avenue des Maquis du Grésivaudan, Grenoble 38043, France.

Published: 23 May 2014

\section{References}

1. De Jonghe B, Sharshar T, Lefaucheur JP, Authier FJ, Durand-Zaleski I, Boussarsar M, Cerf C, Renaud E, Mesrati F, Carlet J, Raphaël JC, Outin H, Bastuji-Garin S, Groupe de Réflexion et d'Etude des Neuromyopathies en Réanimation: Paresis acquired in the intensive care unit: a prospective multicenter study. JAMA 2002, 288:2859-2867

2. Herridge MS, Cheung AM, Tansey CM, Matte-Martyn A, Diaz-Granados N, Al-Saidi F, Cooper AB, Guest CB, Mazer CD, Mehta S, Stewart TE, Barr A, Cook D, Slutsky AS, Canadian Critical Care Trials Group: One-year outcomes in survivors of the acute respiratory distress syndrome. N Engl J Med 2003, 348:683-693.
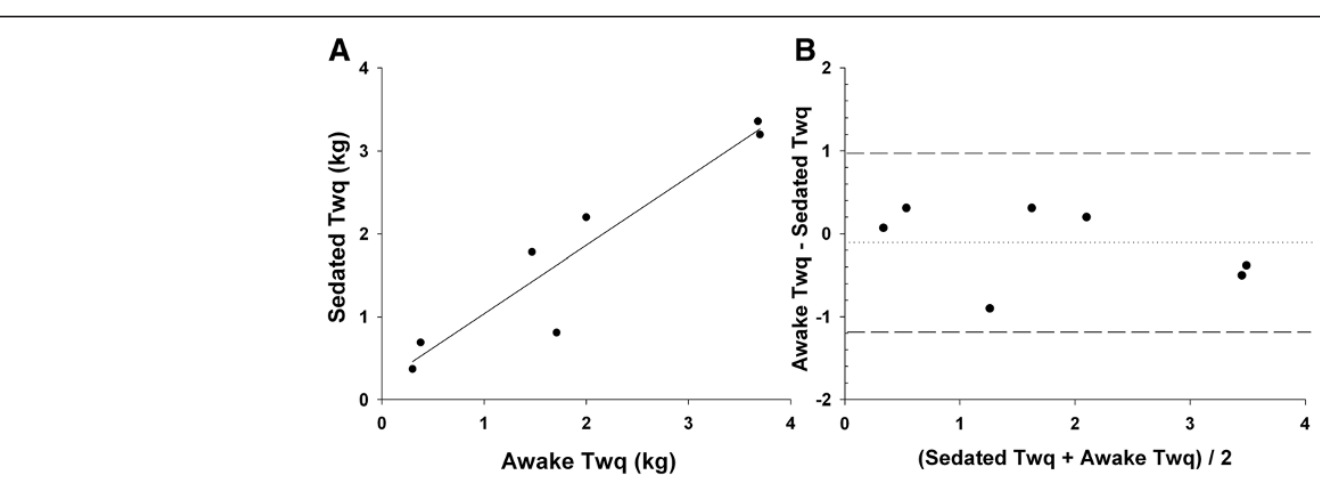

Figure 2 Reproducibility of quadriceps twitch tension (Twq) measurements in sedated versus awake conditions. (A) Linear regression between Twq measured in sedated versus awake conditions in mechanical ventilation patients (Spearman coefficient correlation, $r=0.93$, $P=0.02$ ). (B) Bland-Altman comparison of sedated and awake Twq measurements. Limits of agreement (reference range of differences) were -1.18 and $0.98 \mathrm{~kg}$. The means bias was $-0.13 \mathrm{~kg}$ with a standard deviation of $0.47 \mathrm{~kg}$. 
3. Saey D, Debigare R, LeBlanc P, Mador MJ, Cote CH, Jobin J, Maltais F: Contractile leg fatigue after cycle exercise: a factor limiting exercise in patients with chronic obstructive pulmonary disease. Am J Respir Crit Care Med 2003, 168:425-430.

4. Vivodtzev I, Flore P, Levy P, Wuyam B: Voluntary activation during knee extensions in severely deconditioned patients with chronic obstructive pulmonary disease: benefit of endurance training. Muscle Nerve 2008, 37:27-35.

5. Ginz HF, laizzo PA, Urwyler A, Pargger $H$ : Use of non-invasive-stimulated muscle force assessment in long-term critically ill patients: a future standard in the intensive care unit? Acta Anaesthesiol Scand 2008, 52:20-27.

$10.1186 /$ cc13888

Cite this article as: Vivodtzev et al: Severe and early quadriceps weakness in mechanically ventilated patients. Critical Care 2014, 18:431 